Corresponding author: jonathan.wong@ monashhealth.org

(c) 2020 Wong et al. This article is distributed under the terms of the Creative Commons Attribution-NonCommercial License, which permits reuse and redistribution, except for commercial purposes, provided that the original author and source are credited.

Ontology terms: hematological neoplasm; leukemia; T-cell acute lymphoblastic leukemias

Published by Cold Spring Harbor Laboratory Press

doi:10.1101/mcs.a004994

\section{Failure of tofacitinib to achieve an objective response in a DDX3X-MLLT10 T-lymphoblastic leukemia with activating JAK3 mutations}

\author{
Jonathan Wong, ${ }^{1,2}$ Meaghan Wall, 2,3,4,5 Gregory Philip Corboy, ${ }^{1,2,6}$ \\ Nadine Taubenheim, ${ }^{5,7}$ Gareth Peter Gregory, ${ }^{1,2}$ Stephen Opat, ${ }^{1,2}$ \\ and Jake Shortt ${ }^{1,2}$
}

\begin{abstract}
${ }^{1}$ Department of Hematology, Monash Health, Clayton, 3168, Victoria, Australia; ${ }^{2}$ School of Clinical Sciences, Monash University, Clayton, 3168, Victoria, Australia; ${ }^{3}$ Victorian Cancer Cytogenetics Service, St Vincent's Hospital, Fitzroy, 3065, Victoria, Australia; ${ }^{4}$ St Vincent's Institute, Fitzroy, 3065, Victoria, Australia; ${ }^{5}$ Monash Pathology, Monash Health, Clayton, 3168, Victoria, Australia; ${ }^{6}$ Department of Clinical Pathology, The University of Melbourne, Parkville, 3010, Victoria, Australia; ${ }^{7}$ Center for Cancer Research, Hudson Institute of Medical Research, Clayton, 3168, Victoria, Australia
\end{abstract}

Abstract T-cell lymphoblastic lymphoma/T-cell acute lymphoblastic leukemia (T-LBL/ $T$ ALL) is an aggressive hematological malignancy arising from malignant transformation of T-cell progenitors with poor prognosis in adult patients. Outcomes are particularly dismal in the relapsed/refractory setting, and therapeutic options are limited in this context. Genomic profiling has shown frequent aberrations in the JAK-STAT pathway, including recurrent mutations in JAK3 (15\%-20\% of T-ALL cases), suggesting that JAK kinase inhibition may be a promising therapeutic approach. Activating JAK3 mutations are capable of transforming cytokine-dependent progenitor cells in vitro and causing T-ALL-like disease when expressed in hematopoietic progenitors in vivo. We describe a case of relapsed T-ALL in an adult patient, with two JAK3 activating mutations identified by whole-exome sequencing (WES), leading to hypothesis-based treatment with the JAK1 and JAK3 inhibitor, tofacitinib, following failure of salvage chemotherapy reinduction. Despite the molecularly targeted rationale, tofacitinib did not induce an objective clinical response. Our report suggests that the presence of activating JAK3 mutations does not necessarily confer sensitivity to pharmacological JAK3 inhibition.

[Supplemental material is available for this article.]

\section{INTRODUCTION}

T-cell lymphoblastic lymphoma/T-cell acute lymphoblastic leukemia (T-LBL/T-ALL) is an aggressive hematological malignancy arising from malignant transformation of T-cell progenitors that accounts for $15 \%$ of pediatric and $25 \%$ adult ALL cases (Borowitz et al. 2017). Although childhood T-ALL remission rates and long-term survival have steadily improved, prognosis in adult patients remains poor, particularly in relapsed or refractory cases. Consequently, more effective treatment is urgently needed in this patient group, and recently developed therapies with novel mechanisms of action are currently under investigation.

The DDX3X-MLLT10 fusion is a primary abnormality in T-ALL and cases harboring this fusion cluster within the HOXA subgroup by gene expression profiling (Brandimarte et al. 
2013). Genomic profiling studies in T-ALL have shown frequent aberrations within the Janus kinase (JAK)-signal transducer and activator of transcription (STAT) pathway, such as recurrent mutations in JAK1 and JAK3, suggesting that JAK kinase inhibition may be a promising therapeutic approach. JAK1/JAK3 mutations are recurrent and enriched in the HOXA subgroup and in patients with MLLT10 gene rearrangements, but JAK1/JAK3 mutations do not appear to have been described previously in DDX3X-MLLT10 T-ALL (Liu et al. 2017). JAK3 mutations are present in 15\%-20\% of T-ALL cases (Bains et al. 2012; De Keersmaecker et al. 2012; Kalender Atak et al. 2012, 2013; Zhang et al. 2012; Yin et al. 2014; Greenplate et al. 2018). Activating (gain-of-function) JAK3 mutations induce constitutive JAK activation and STAT phosphorylation and have also been identified in other hematological malignancies including T-cell and myeloid neoplasms (Cornejo et al. 2009). Mutant JAK3 is capable of transforming cytokine-dependent progenitor cells in vitro and causing T-ALL-like disease when expressed in hematopoietic progenitors in vivo (Degryse et al. 2014).

Here, we report the identification of two JAK3 activating mutations by whole-exome sequencing (WES) in a patient presenting with relapsed T-ALL with DDX3X-MLLT10 fusion, leading to hypothesis-based treatment with the JAK1 and JAK3 inhibitor, tofacitinib. Despite the molecularly targeted rationale, tofacitinib did not induce an objective clinical response. We conclude that the presence of activating JAK3 mutations does not necessarily evoke sensitivity to pharmacological JAK3 inhibition.

\section{RESULTS}

\section{Clinical Presentation}

A 69-yr-old woman presented with exertional dyspnea and pruritus. A mediastinal mass was identified on computerized tomography (CT) imaging.

Transbronchial cryobiopsy of the mediastinal mass identified diffuse sheets of intermediate-sized, atypical lymphoid cells with minimal cytoplasm and enlarged hyperchromatic nuclei, expressing CD3, CD4, CD7, CD10, and TdT by immunohistochemical staining, consistent with a diagnosis of T-lymphoblastic lymphoma (T-LBL). Fluorodeoxyglucose positron emission tomography (FDG-PET) imaging confirmed nodal disease limited to the mediastinum and left hilum, in keeping with Ann Arbor stage II. There was no evidence of extranodal involvement on bone marrow biopsy and magnetic resonance imaging of the central nervous system. She commenced treatment with HyperCVAD/methotrexatecytarabine induction chemotherapy (Kantarjian et al. 2004) and achieved complete structural and metabolic remission after four cycles, followed by consolidative radiotherapy to the mediastinal mass. Allogeneic stem cell transplant was discussed with the patient at this point but was not pursued on the basis of her advanced age and treatment preferences.

The patient presented eight months later with fatigue. Full blood examination revealed circulating medium-sized blasts comprising $81 \%$ of nucleated cellularity accompanied by pancytopenia: hemoglobin $6.4 \mathrm{~g} / \mathrm{dL}$, absolute neutrophil count $0.01 \times \mathrm{k} / \mu \mathrm{L}$, platelet count $9 \times \mathrm{k} / \mu \mathrm{L}$. A bone marrow aspirate and trephine biopsy were obtained for morphologic assessment, flow cytometry, fluorescence in situ hybridization (FISH), and chromosome studies. The marrow was markedly hypercellular (90\%) and predominantly composed of lymphoblasts (90\%-95\% of marrow cellularity) with reduced trilineage hematopoiesis (Fig. 1A). Flow cytometry of the bone marrow aspirate demonstrated a blast population comprising $99 \%$ of leukocytes, which expressed the following antigens: CD1a (partial dim), cCD3, CD4 (dim), CD5 (dim), CD7, CD10, and TdT. Blasts were negative for CD19 and MPO. The morphologic and immunophenotypic features confirmed relapsed T-ALL. 
COLD SPRING HARBOR Molecular Case Studies
Tofacitinib in JAK3-mutated T-lymphoblastic leukemia
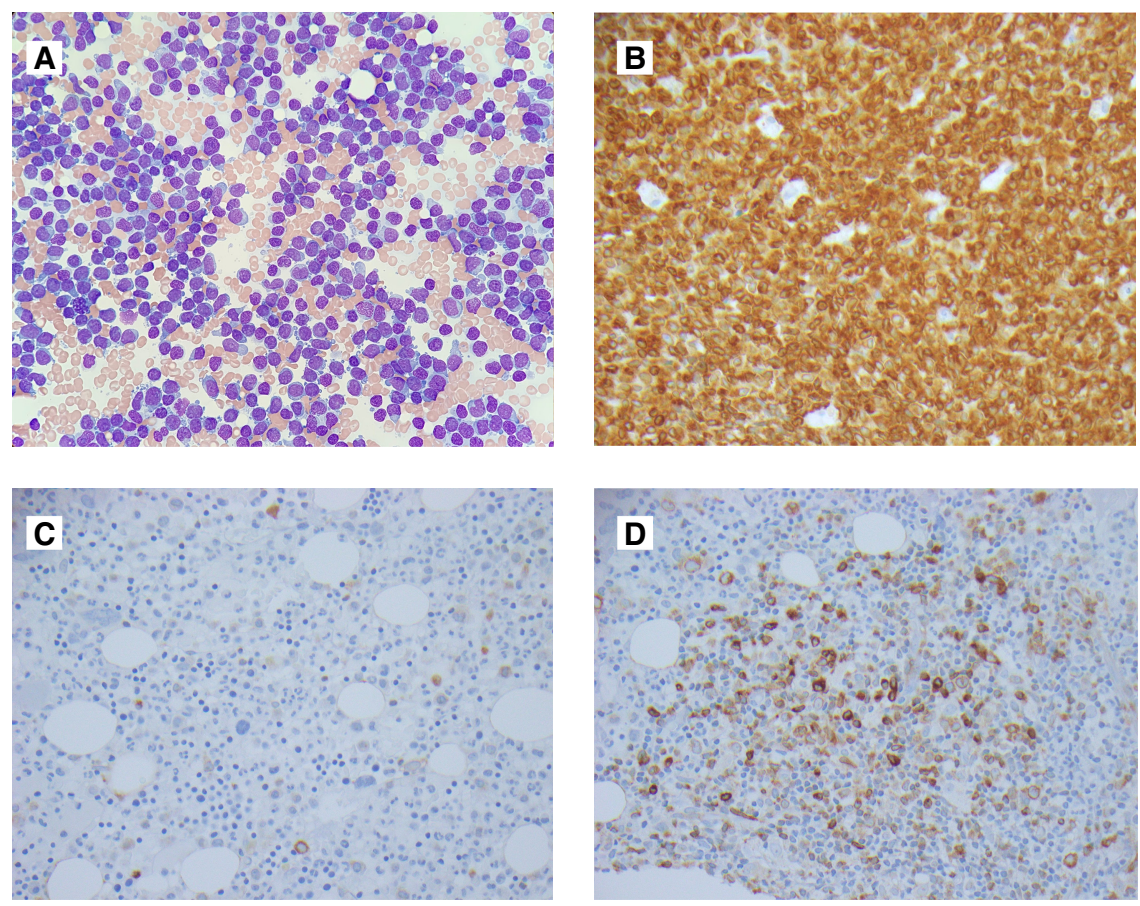

Figure 1. (A) Routinely stained bone marrow aspirate specimen obtained at relapse demonstrates marked hypercellularity predominantly comprised of medium-sized blasts. Magnification, 40×. (B-D) Immunohistochemical stain performed with anti-BCL2 antibody (clone SP66, Ventana Roche) on formalin-fixed, paraffin-embedded tissue of bone marrow core biopsy specimen obtained at relapse demonstrates uniformly strong BCL2 expression in leukemic blasts $(B)$; normal bone marrow core biopsy specimen as control $(C)$; bone marrow core biopsy specimen with follicular non-Hodgkin lymphoma demonstrating BCL2 positive lymphoma cells (D). Magnification, 40x.

\section{Treatment Outcomes}

The patient commenced FLAG (fludarabine, cytarabine, filgrastim) salvage chemotherapy (Montillo et al. 2009). Her treatment was complicated by febrile neutropenia attributable to a urinary tract infection. Despite intensive chemotherapy, the patient failed to clear blasts from her peripheral blood, which were confirmed to be T-ALL by immunophenotyping. At this point, no clinical trials were available and it was deemed futile to administer further intensive chemotherapy. Given the finding of activating JAK3 mutations identified on sequencing at diagnosis and at relapse (see Methods section and Tables 1 and 2), we hypothesized that JAK-inhibition with tofacitinib might rationally evoke an anti-tumor response. Tofacitinib (marketed as Xeljanz) has potent inhibitory activity against JAK1 and JAK3 (Flanagan et al. 2010; Meyer et al. 2010). We posited that this was preferable to the more JAK2-selective agent, ruxolitinib, which does not inhibit JAK3 activity. The patient was informed that tofacitinib treatment was not approved for leukemia treatment and consented to a trial of therapy after considering the potential risks. Tofacitinib was supplied under a Pfizer non-commercial supply compassionate access program following notification of the Monash Health Drug and Therapeutic Committee and Australian Therapeutic Goods Administration. She commenced treatment with tofacitinib at a dose of $5 \mathrm{mg}$ BD on Day 17 post-FLAG. A bone marrow aspirate was performed on Day 21 post-FLAG, which showed persistent marrow infiltration with blasts ( $97 \%$ of nucleated cellularity). She started weekly oral dexamethasone $40 \mathrm{mg}$ and continued on tofacitinib. In the absence of any further evidence of an objective response, tofacitinib was ceased from day 36 post-FLAG (Fig. 2). 


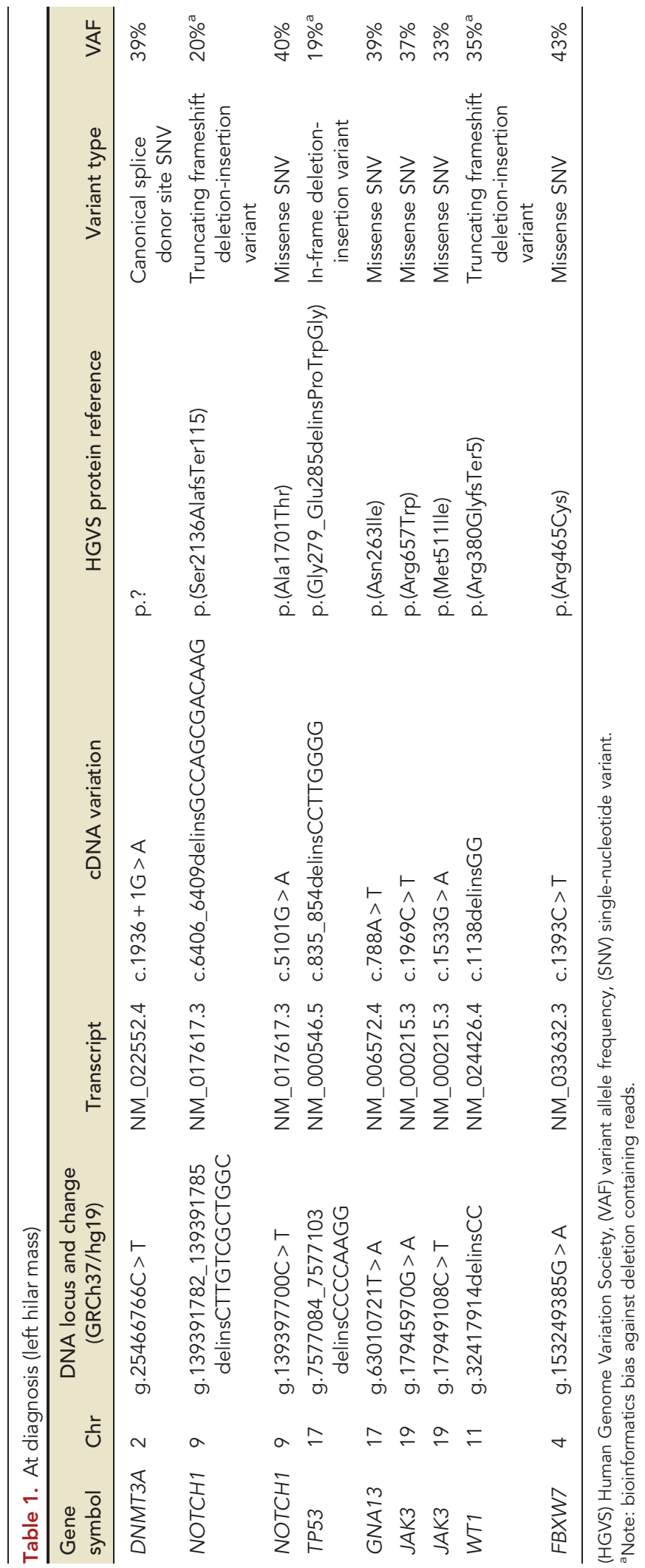




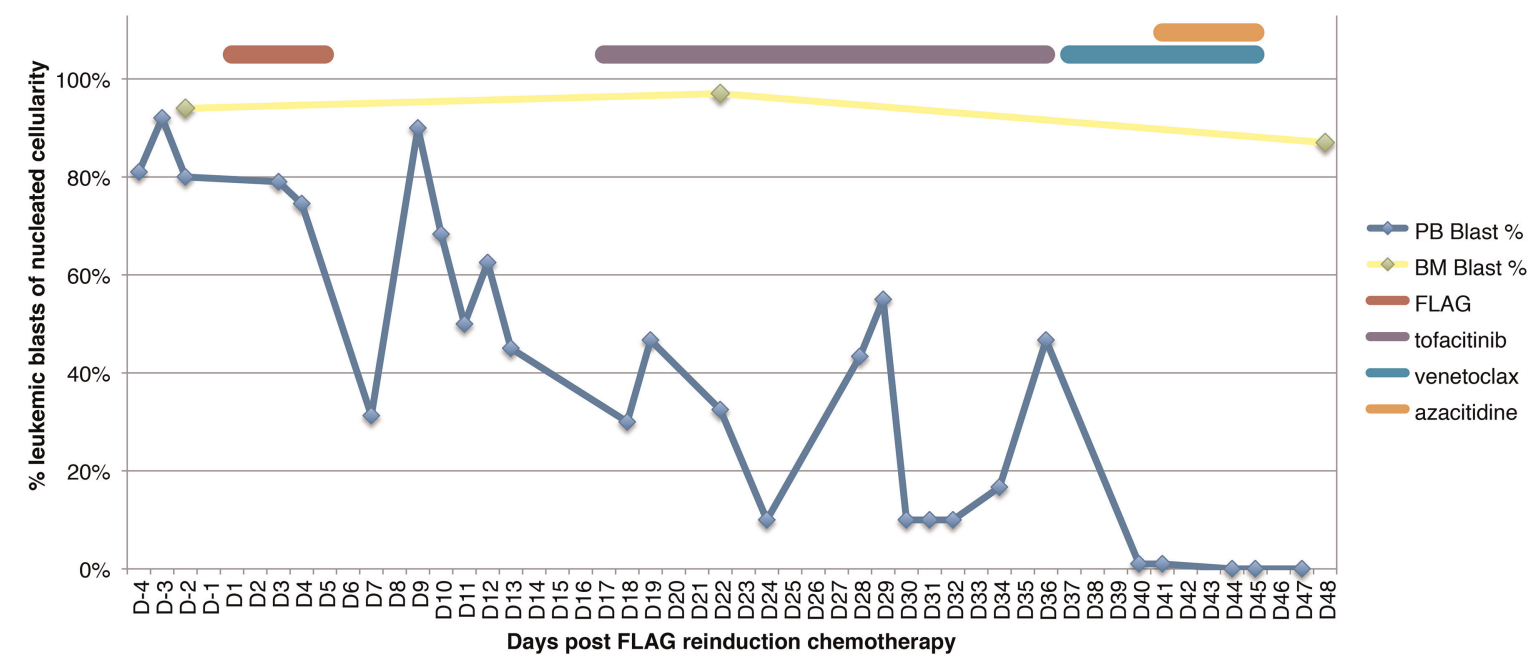

Figure 2. Timeline of treatment and peripheral blood leukemic blast percentages. (BM) Bone marrow, (D) day, (FLAG) fludarabine, cytarabine, filgrastim (G-CSF), (PB) peripheral blood.

Mutations in DNA-methylation regulators (e.g., DNMT3A, TET2) induce epigenetic changes that may be modulated by DNA-methyltransferase inhibitors such as decitabine and azacitidine. In particular, TET2 mutations predict a higher response rate to hypomethylating agents in myelodysplasia (Bejar et al. 2014). JAK-STAT pathway activation increases anti-apoptotic BCL2 family protein expression in lymphoid cancer (Waibel et al. 2014), and we noted high BCL2 expression in our patient's bone marrow trephine (Fig. 1B). Moreover, combination therapy with decitabine and the BCL2 inhibitor, venetoclax, was recently reported to induce clinical remission in another patient with relapsed and refractory T-ALL (Rahmat et al. 2018).

Having received a cycle of azacitidine, venetoclax was obtained through the AbbVie compassionate access program and commenced at $100 \mathrm{mg}$ daily. There was no evidence of tumor lysis syndrome, but venetoclax dose escalation was not attempted as the patient was receiving posaconazole antifungal prophylaxis (which inhibits venetoclax metabolism). A second cycle of azacitidine was commenced on Day 40 post-FLAG. At this point there had still been no objective evidence of an antileukemic response and the patient developed febrile neutropenia with significant clinical deterioration. A further bone marrow biopsy (Day 54 post-FLAG) showed ongoing leukemia with $87 \%$ blasts. In view of her poor response to treatment, the decision was made to withdraw active treatment and the patient died shortly after this.

\section{DISCUSSION}

Disease-free survival is poor in adults with a diagnosis of T-ALL, particularly when compared to pediatric T-ALL patients. Outcomes are particularly dismal in the relapsed/refractory setting, and $<10 \%$ of patients survive long-term (Marks and Rowntree 2017). There are limited therapeutic options available in this context. Here, we describe a patient initially diagnosed with mediastinal T-LBL who achieved remission with induction chemotherapy but relapsed with T-ALL within eight months. WES on the diagnostic biopsy specimen identified two JAK3 hotspot missense variants, p.Met511lle and p.Arg657Trp, both previously established as activating mutations (Degryse et al. 2014). Targeted gene sequencing on the bone 
marrow aspirate specimen at relapse confirmed the persistence of these mutations. Subsequently, targeted JAK-STAT pathway inhibition with tofacitinib was introduced following salvage chemotherapy reinduction.

Interleukin 7 (IL7) signaling is essential for normal T-cell development and plays a major role in differentiation and homeostasis. IL7 interacts with heterodimeric IL7 receptor (IL7R), which induces reciprocal JAK1 and JAK3 phosphorylation and subsequent recruitment and activation of STAT5. Phosphorylated STAT5 dimerizes and translocates to the nucleus where it regulates gene transcription (e.g., BCL2 family genes) (Waibel et al. 2014). Unlike other JAK family kinases, JAK3 expression and function is mostly restricted to the hematopoietic compartment (Cornejo et al. 2009; Degryse and Cools 2015; Girardi et al. 2017).

Activating mutations in this pathway (ILR7, JAK1, JAK3, STAT5) are present in 20\%-30\% of T-ALL cases (Zhang et al. 2012; Vicente et al. 2015; Girardi et al. 2017). JAK3 mutations are most frequently seen (Bains et al. 2012; De Keersmaecker et al. 2012; Kalender Atak et al. 2012, 2013; Zhang et al. 2012; Yin et al. 2014; Greenplate et al. 2018). Functional studies investigating the oncogenic role of JAK3 in T-ALL have shown the JAK3 p.Met511lle pseudokinase domain mutant to be the most efficient oncokinase with the highest transforming properties (Yamashita et al. 2010). In vivo data support the transforming role of mutant JAK3 in a mouse bone marrow transplant model of JAK3-induced T-ALL (Degryse et al. 2014). One-third of JAK3-mutated T-ALL cases harbor two JAK3 mutations, which can be monoallelic or less frequently biallelic (Degryse et al. 2018). JAK3 p.Met511lle increases oncogenic potential via acquisition of additional mutations in the mutant JAK3 allele (Degryse et al. 2018). Double JAK3 mutants show increased STAT5 activation compared to single JAK3 mutants, suggesting that progression of JAK3 mutant T-ALL cases may be associated with acquisition of additional JAK3 mutations and subsequent increase in JAK/STAT signaling (Degryse et al. 2018).

In our patient, all variants identified by WES demonstrated concordant allele frequency, including both JAK3 variants, suggesting they were present in the same leukemic cells, rather than separate clones. The p.Met511lle and p.Arg657Trp variants had concordant variant allele frequencies (VAFs) of $31 \%$ and $37 \%$, respectively, at diagnosis, and concordant VAFs of $97 \%$ and $93 \%$, respectively in the relapsed leukemia sample, suggesting that both variants are likely on the same allele with either copy-neutral loss of heterozygosity of 19p13 or deletion of the second JAK3 allele. The two JAK3 variants are $436 \mathrm{bp}$ apart and are too far apart to phase on short-read sequencing. Large-fragment amplification of JAK3 encompassing both variants was performed to experimentally validate the hypothesis that both variants were present on the same allele, but was unsuccessful.

Loss-of-function mutations affecting JAK3 signaling in patients with severe combined immunodeficiency disease have been shown to severely impair lymphoid development (Pesu et al. 2005). On this basis, the potential therapeutic effect of JAK3 inhibition has been investigated as targeted immunosuppression for prevention of solid organ graft rejection and in autoimmune inflammatory diseases such as rheumatoid arthritis. A number of JAK inhibitors have been studied, with variable selectivity against JAK3 compared to other JAK family members. Originally described as a selective JAK3 inhibitor, tofacitinib also inhibits JAK1 and its efficacy may be due to the combined inhibition of both kinases (Thoma et al. 2014). Tofacitinib has received U.S. Federal Drug Administration (FDA) approval for the treatment of refractory rheumatoid arthritis.

Different activating mutations of JAK3 confer differential sensitivity to JAK inhibitors (Losdyck et al. 2015; Steven Martinez et al. 2016). Preclinical data suggest that most, but not all, JAK3 mutants transform T-ALL cell lines to cytokine-independent proliferation in vitro and cause leukemia in vivo. However, JAK3 pseudokinase domain mutants (including p.Met511 lle and p.Arg657Trp mutants, identified in our patient) require JAK1 for their transforming potential. In contrast, JAK3 kinase domain mutations can induce cell transformation 
independent from JAK1. In line with these findings, JAK3 pseudokinase domain mutants demonstrated more sensitivity to ruxolitinib (a JAK1/JAK2 inhibitor) than JAK3 kinase domain mutants, whereas both JAK3 pseudokinase and kinase domain mutants were sensitive to tofacitinib. Tofacitinib and ruxolitinib demonstrated synergistic inhibition in JAK3 pseudokinase domain mutants (Degryse et al. 2014). The effect of double JAK3 mutants on sensitivity to JAK inhibitors is unclear. Degryse and colleagues identified two cases of p.Met511lle and p.Arg657Trp double mutants but did not test sensitivity to ruxolitinib (Degryse et al. 2018). To the best of our knowledge, there are no reports of tofacitinib sensitivity testing for this double mutant so it is unknown if tofacitinib is active against the double mutant.

Activating JAK3 mutations have been identified in other T-cell malignancies and are seen most frequently in T-cell prolymphocytic leukemia (T-PLL) at a rate of 30\%-40\% (Bellanger et al. 2013; Bergmann et al. 2014; Kiel et al. 2014; Stengel et al. 2015; López et al. 2016). Targeted JAK3 inhibition with tofacitinib has elicited responses in several cases of T-PLL, both as a single agent (Li et al. 2017) and in combination with ruxolitinib (GomezArteaga et al. 2019). Interestingly, both of these cases harbored the JAK3 hotspot activating mutation, p.Met511lle, as well as a second JAK3 missense mutation in each case (p.Ala573Val pseudokinase domain mutation and p.Leu875His kinase domain mutation, respectively). JAK3 mutations are not common in T-cell large granular lymphocytic leukemia (T-LGLL), but a proportion of patients demonstrate activating STAT3 mutations; in a cohort of nine patients with T-LGLL (four harboring STAT3 mutations), tofacitinib induced a hematologic response in six patients (Bilori et al. 2015).

To our knowledge, this is the first report describing tofacitinib in a patient with T-ALL harboring gain-of-function JAK3 mutations. We cannot conclude that the tofacitinib exposure did not have a cytostatic (rather than apoptotic) effect, but no significant clinical activity was observed either as a single agent or when combined with dexamethasone. It is possible that therapeutic levels of tofacitinib were not achieved, although this seems unlikely as the patient did not have any signs or symptoms of malabsorption and was taking concurrent potent cytochrome 4550 inhibitors (i.e., posaconazole). In a preclinical mouse model, in vivo response was observed within 6 days of commencing treatment with tofacitinib, suggesting sufficient duration of exposure to tofacitinib in our patient (Degryse et al. 2014). This case may indicate that established leukemia may become independent of JAK3 signaling or that unimpeded JAK2 signaling was a pro-survival mechanism in this case. Indeed, one report evaluating the ex vivo sensitivity of T-PLL patient samples to JAK inhibitors showed poor correlation between specific mutations in the JAK-STAT pathway and response (Andersson et al. 2017). Furthermore, in a preclinical model, pSTAT5 expression in M511I + A573V and M511I + A572T double mutants in Ba/F3 were higher than PSTAT5 expression in single mutants, so it is possible that drug concentrations may have been inadequate for inhibition of JAK-STAT signaling in the presence of JAK3 double mutants (Degryse et al. 2018).

The role of other mutations in potential resistance to tofacitinib is speculative. The TP53 mutation variant allele frequency increased between diagnosis and relapse from 19\% (Table 1) to $86 \%$ (Table 2), consistent with copy-neutral loss of heterozygosity (CN-LOH) or deletion of the second allele. TP53 loss-of-function mutation is known to drive resistance to DNA damaging agents and is a predictor of poor prognosis in adults treated with chemotherapy, but its impact on response to tofacitinib remains unknown (Diccianni et al. 1994; Hof et al. 2011). However, Tp53 null mice with B-ALL and oncogene addiction to JAK-STAT signaling still responded to tofacitinib and ruxolitinib (Cheng et al. 2016). This would indicate that responses to JAK inhibition may be agnostic to p53 status; however, more functional studies and preclinical models are needed.

JAK-STAT pathway activation has been shown to up-regulate anti-apoptotic BCL2 family protein expression in lymphoid cancer (Waibel et al. 2014). In this case, high BCL2 protein 
expression was seen on immunohistohemical staining of the patient's bone marrow trephine (Fig. 1B). Venetoclax (ABT-199) is a highly specific BCL-2 inhibitor. Preclinical studies have shown in vitro and in vivo sensitivity of some T-ALL cell lines to venetoclax, either alone or in combination with other agents (Peirs et al. 2014). A report of venetoclax combined with low-intensity chemotherapy reported a durable response in one patient with early $\mathrm{T}$-cell precursor ALL, and a complete but transient response in one patient with T-ALL (Numan et al. 2018). This was consistent with preclinical data demonstrating particular susceptibility of ETP-ALL to BCL2 inhibition, because of lesser dependence on BCL-XL compared to more mature subtypes of T-ALL (Chonghaile et al. 2014). In our case there was no apparent response to venetoclax, indicating BCL2-independent pro-survival mechanisms that could be explained by the up-regulation of BCL-XL in JAK-STAT dysregulated neoplasms (Waibel et al. 2014). Although it is possible the lack of efficacy of venetoclax could be attributable to underdosing, it was postulated that there would be a p450 interaction with concurrent posaconazole administration, reducing the metabolism of venetoclax and thereby increasing the therapeutic level. The patient's limited exposure to azacitidine (only two cycles) makes it inappropriate to speculate as to whether ongoing hypomethylating agent treatment in combination with venetoclax might have yielded a disease response.

Although this case report indicates that activating JAK3 mutations do not necessarily sensitize to tofacitinib therapy, further preclinical and clinical exploration of JAK inhibition in JAK-STAT dysregulated acute leukemia is clearly warranted. Furthermore, gene expression data and pathway analysis would potentially have assisted with targeted approaches to management in this case, supporting the role of robust and representative assays in clinical management.

\section{METHODS}

WES with targeted analysis of genes related to lymphoid malignancies was performed on DNA extracted from the initial diagnostic left hilar lung mass cryobiopsy and genomic DNA derived from buccal swab normal specimens. Tumor purity was estimated as $90 \%$, assessed on hematoxylin and eosin-stained tissue obtained by cryobiopsy. Libraries were prepared using Agilent Clinical Research Exome 1 (CRE1) (Agilent) followed by massively parallel sequencing using a HiSeq 3000 instrument (Illumina) with 100-bp paired-end reads. Tumor-normal comparison to generate somatic variant calls was conducted using the Seqliner bioinformatic pipeline (http://seqliner.org). Mean target region sequencing coverage was 140× (tumor) and 60× (germline) (Supplemental Table S1).

A list of target genes was selected following a review of the literature regarding recurrent mutations in lymphoid malignancies, performed by three independent contributors (Supplemental Table S2). After filtering, seven nonsynonymous somatic variants presumed to be functionally important were identified in the tumor: five single-nucleotide variants (SNVs) and two short insertions and deletions (indels) involving DNMT3A, GNA13, JAK3, NOTCH1, and TP53 (Table 1). We identified two JAK3 hotspot missense SNVs: NM_ 000215.3: c.1533G > A, p.Met511lle and NM_000215.3: c.1969C > T, p.Arg657Trp. Both variants occur in the pseudokinase (JH2) domain (Fig. 3) and have been established as activating mutations in JAK3 (Degryse et al. 2014). The two JAK3 variants are $436 \mathrm{bp}$ apart and were thus too far apart to phase on short-read sequencing. Large-fragment amplification of JAK3 encompassing both variants was unsuccessful.

Genomic studies were performed on the bone marrow aspirate specimen taken at relapse. Conventional chromosome studies showed the following karyotype: $46, X, t(X ; 10 ; 2)$ (p11.4;p12;q31),del(9)(p?13p?21)[7]/47,idem,+20[4]/46, idem, ?t(4;14)(q13;q21),t(12;22)(p12; $q 11.2)[7] / 46, X, \operatorname{der}(X) t(X ; 2)(p 11.4 ; q 31), \operatorname{der}(1) t(1 ; 2)(q 21 ; p 23), \operatorname{der}(2) t(1 ; 2)(q 21 ; p 23) t(2 ; 10)(q 31 ;$ 
COLD SPRING HARBOR Molecular Case Studies
Tofacitinib in JAK3-mutated T-lymphoblastic leukemia

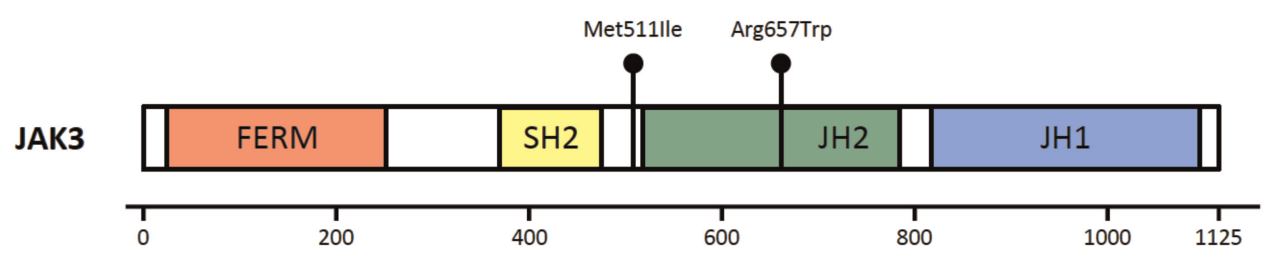

Figure 3. Missense mutations in JAK3 identified in our patient. Schematic shows JAK3 protein domain structures. Amino acid numbers are shown below. Missense mutations are denoted by circles with codon changes shown. (FERM) The conserved domain named for its founding members (band 4.2, exrin, radixin, and moesin), (JH1) JAK homology domain 1, the functional kinase domain, (JH2) JAK homology domain 2, also known as the pseudokinase domain, (SH2) Src homology domain 2.

p12), $\operatorname{add}(3)(p 13), \operatorname{add}(5)(q 13), \operatorname{add}(9)(q 22), \operatorname{del}(9)(p ? 13 p ? 21), \operatorname{der}(10) t(X ; 10)(p 11.4 ; p 12),-13,-13$, +mar1,+mar2[3] (Table 2). This identified a three-way $X ; 10 ; 2$ translocation and deletion of part of $9 p$ in all 22 metaphases analyzed. Multiple subclones were present. Four metaphases also showed trisomy 20, whereas another seven contained reciprocal 4;14 and 12;22 translocations. Three metaphases were more complex. They showed a reciprocal 1;2 translocation involving $1 q$ and the short arm of the derivative chromosome 2 , abnormalities of $3 p$, $5 q, 9 q$, and apparent nullisomy 13 with two marker chromosomes. The $t(X ; 10 ; 2)$ appeared to be a variant of the $\mathrm{X} ; 10$ translocation that forms a DDX3X-MLLT10 gene fusion. The $t(X ; 10)$ is a rare but recurrent translocation in T-LBL/T-ALL and has an association with NOTCH1 mutation and deletion of 9p (Brandimarte et al. 2014).

Targeted massively parallel sequencing of 76 genes with diagnostic, prognostic, and therapeutic relevance in hematological malignancies was performed on DNA derived from the bone marrow aspirate specimen obtained at time of relapse. Libraries were prepared using the KAPA Hyper Prep Kit (KAPA Biosystems) and then enriched using a SureSelectXT Custom Capture Panel (Agilent) and sequenced on a NextSeq 500 instrument (Illumina) with 75-bp paired-end reads. This confirmed the presence of the variants identified on WES of the initial diagnostic biopsy, including the two JAK3 hotspot missense variants (Table 2). Three additional variants were identified. Two variants were in genes not included in the target gene review list for exome analysis: a WT1 frameshift mutation, NM_024426.4: c.1138delinsGG, p.Arg380Glyfs*5, and a FBXW7 hotspot missense mutation, NM_ 033632.3: c.1393C > T, p.Arg465Cys. However, retrospective review of the exome sequencing data showed that both WT1 and FBXW7 mutations were present in the diagnostic sample (Table 1). A third NOTCH1 variant was also identified, an in-frame insertion, NM_017617.3: c.5154_5155insCGC, p.lle1718_Glu1719insArg. This variant was not present on WES performed on the initial diagnostic specimen.

\section{ADDITIONAL INFORMATION}

\section{Data Deposition and Access}

The variants were submitted to COSMIC (https://cancer.sanger.ac.uk/cosmic) and can be found under accession number COSP48326. Patient consent was not obtained for deposition of raw sequencing data. The Melbourne Genomics Health Alliance can be contacted for anonymized cohort data release via their data release program.

\section{Ethics Statement}

The whole-exome sequencing performed in this study was approved by the Melbourne Health Human Research Ethics Committee as an amendment to the Melbourne Genomics Protocol Number 2013.245. 
Competing Interest Statement

The authors have declared no competing interest.

Received November 19, 2019; accepted in revised form June 5, 2020.
Informed consent was obtained in writing from the patient prior to collection of blood, tumor, and buccal swab samples. On enrollment into the study, the patient signed a patient information and consent form approved by central and local human research ethics committees. The consent included use of patient data for publication. Postmortem consent for publication was also obtained from the deceased patient's next of kin.

The authors report no pertinent disclosures, and no conflict of interest concerning the materials or methods used in this study or findings described in this paper.

\section{Author Contributions}

J.W. acquired clinical data and images, created the table and figures, and developed the manuscript with the assistance of J.S. G.P.C. performed and interpreted whole-exome sequencing analysis. N.T. performed targeted JAK3 sequencing analysis. M.W. performed and interpreted conventional chromosome analysis. G.P.G. and S.O. supervised the whole-exome sequencing project. All authors critically edited and approved the final manuscript.

\section{Funding}

The Melbourne Genomics Health Alliance is supported financially by the State Government of Victoria (Department of Health and Human Services) and by its member institutions. J.W. was supported by a Monash Health Emerging Researcher Fellowship.

\section{REFERENCES}

Andersson El, Pützer S, Yadav B, Dufva O, Khan S, He L, Sellner L, Schrader A, Crispatzu G, Oleś M, et al. 2017. Discovery of novel drug sensitivities in T-PLL by high-throughput ex vivo drug testing and mutation profiling. Leukemia 32: 774-787. doi:10.1038/leu.2017.252

Bains T, Heinrich MC, Loriaux MM, Beadling C, Nelson D, Warrick A, Neff TL, Tyner JW, Dunlap J, Corless CL, et al. 2012. Newly described activating JAK3 mutations in T-cell acute lymphoblastic leukemia. Leukemia 26: 2144-2146. doi:10.1038/leu.2012.74

Bejar R, Lord A, Stevenson K, Bar-Natan M, Pérez-Ladaga A, Zaneveld J, Wang H, Caughey B, Stojanov P, Getz $\mathrm{G}$, et al. 2014. TET2 mutations predict response to hypomethylating agents in myelodysplastic syndrome patients. Blood 124: 2705-2712. doi:10.1182/blood-2014-06-582809

Bellanger D, Jacquemin V, Chopin M, Pierron G, Bernard OA, Ghysdael J, Stern M-H. 2013. Recurrent JAK1 and JAK3 somatic mutations in T-cell prolymphocytic leukemia. Leukemia 28: 417-419. doi:10.1038/leu .2013 .271

Bergmann AK, Schneppenheim S, Seifert M, Betts MJ, Haake A, López C, Maria Murga Penas E, Vater I, Jayne S, Dyer MJS, et al. 2014. Recurrent mutation of JAK3 in T-cell prolymphocytic leukemia. Genes Chromosomes Cancer 53: 309-316. doi:10.1002/gcc.22141

Bilori B, Thota S, Clemente MJ, Patel B, Jerez A, Afable M II, Maciejewski JP. 2015. Tofacitinib as a novel salvage therapy for refractory T-cell large granular lymphocytic leukemia. Leukemia 29: 2427-2429. doi:10 .1038/leu.2015.280

Borowitz MJ, Chan JKC, Béné M-C, Arber DA. 2017. WHO classification of tumours of haematopoietic and lymphoid tissues. In T-lymphoblastic leukaemia/lymphoma (ed. Swerdlow SH, Campo E, Harris NL, et al.), pp. 209-212. IARC, Lyon.

Brandimarte L, Pierini V, Di Giacomo D, Borga C, Nozza F, Gorello P, Giordan M, Cazzaniga G, Kronnie Te G, La Starza $R$, et al. 2013. New MLLT10 gene recombinations in pediatric T-acute lymphoblastic leukemia. Blood 121: 5064-5067. doi:10.1182/blood-2013-02-487256

Brandimarte L, La Starza R, Gianfelici V, Barba G, Pierini V, Di Giacomo D, Cools J, Elia L, Vitale A, Luciano L, et al. 2014. DDX3X-MLLT10 fusion in adults with NOTCH1 positive T-cell acute lymphoblastic leukemia. Haematologica 99: 64-66. doi:10.3324/haematol.2013.101725

Cheng Y, Chikwava K, Wu C, Zhang H, Bhagat A, Pei D, Choi JK, Tong W. 2016. LNK/SH2B3 regulates IL-7 receptor signaling in normal and malignant B-progenitors. J Clin Invest 126: 1267-1281. doi:10.1172/ $\mathrm{JCl} 81468$

Chonghaile TN, Roderick JE, Glenfield C, Ryan J, Sallan SE, Silverman LB, Loh ML, Hunger SP, Wood B, DeAngelo DJ, et al. 2014. Maturation stage of T-cell acute lymphoblastic leukemia determines BCL-2 
versus BCL-XL dependence and sensitivity to ABT-199. Cancer Discov 4: 1074-1087. doi:10.1158/21598290.CD-14-0353

Cornejo MG, Boggon TJ, Mercher T. 2009. JAK3: a two-faced player in hematological disorders. Int J Biochem Cell Biol 41: 2376-2379. doi:10.1016/j.biocel.2009.09.004

Degryse S, Cools J. 2015. JAK kinase inhibitors for the treatment of acute lymphoblastic leukemia. J Hematol Oncol 8: 91. doi:10.1186/s13045-015-0192-7

Degryse S, de Bock CE, Cox L, Demeyer S, Gielen O, Mentens N, Jacobs K, Geerdens E, Gianfelici V, Hulselmans $\mathrm{G}$, et al. 2014. JAK3 mutants transform hematopoietic cells through JAK1 activation, causing T-cell acute lymphoblastic leukemia in a mouse model. Blood 124: 3092-3100. doi:10.1182/blood-201404-566687

Degryse S, Bornschein S, de Bock CE, Leroy E, Vanden Bempt M, Demeyer S, Jacobs K, Geerdens E, Gielen O, Soulier J, et al. 2018. Mutant JAK3 signaling is increased by loss of wild-type JAK3 or by acquisition of secondary JAK3 mutations in T-ALL. Blood 131: 421-425. doi:10.1182/blood-2017-07-797597

De Keersmaecker K, Atak ZK, Li N, Vicente C, Patchett S, Girardi T, Gianfelici V, Geerdens E, Clappier E, Porcu $\mathrm{M}$, et al. 2012. Exome sequencing identifies mutation in CNOT3 and ribosomal genes RPL5 and RPL10 in T-cell acute lymphoblastic leukemia. Nat Genet 45: 186-190. doi:10.1038/ng.2508

Diccianni MB, Yu J, Hsiao M, Mukherjee S, Shao LE, Yu AL. 1994. Clinical significance of p53 mutations in relapsed T-cell acute lymphoblastic leukemia. Blood 84: 3105-3112. doi:10.1182/blood.V84.9.3105 .3105

Flanagan ME, Blumenkopf TA, Brissette WH, Brown MF, Casavant JM, Shang-Poa C, Doty JL, Elliott EA, Fisher MB, Hines M, et al. 2010. Discovery of CP-690,550: a potent and selective janus kinase (JAK) inhibitor for the treatment of autoimmune diseases and organ transplant rejection. J Med Chem 53: 8468-8484. doi:10 $.1021 / \mathrm{jm} 1004286$

Girardi T, Vicente C, Cools J, De Keersmaecker K. 2017. The genetics and molecular biology of T-ALL. Blood 129: 1113-1123. doi:10.1182/blood-2016-10-706465

Gomez-Arteaga A, Margolskee E, Wei MT, Koen vB, Inghirami G, Horwitz S. 2019. Combined use of tofacitinib (pan-JAK inhibitor) and ruxolitinib (a JAK1/2 inhibitor) for refractory T-cell prolymphocytic leukemia (T-PLL) with a JAK3 mutation. Leukemia Lymphoma 33: 1-6. doi:10.1080/10428194.2019.1594220

Greenplate A, Wang K, Tripathi RM, Palma N, Ali SM. 2018. Genomic profiling of T-cell neoplasms reveals frequent JAK1 and JAK3 mutations with clonal evasion from targeted therapies. JCO Precis Oncol 2018: 1-16. doi:10.1200/PO.17.00019

Hof J, Krentz S, van Schewick C, Korner G, Shalapour S, Rhein P, Karawajew L, Ludwig W-D, Seeger K, Henze G, et al. 2011. Mutations and deletions of the TP53 gene predict nonresponse to treatment and poor outcome in first relapse of childhood acute lymphoblastic leukemia. J Clin Oncol 29: 3185-3193. doi:10.1200/ JCO.2011.34.8144

Kalender Atak Z, De Keersmaecker K, Gianfelici V, Geerdens E, Vandepoel R, Pauwels D, Porcu M, Lahortiga I, Brys V, Dirks WG, et al. 2012. High accuracy mutation detection in leukemia on a selected panel of cancer genes. PLoS ONE 7: e38463-11. doi:10.1371/journal.pone.0038463

Kalender Atak Z, Gianfelici V, Hulselmans G, De Keersmaecker K, Devasia AG, Geerdens E, Mentens N, Chiaretti S, Durinck K, Uyttebroeck A, et al. 2013. Comprehensive analysis of transcriptome variation uncovers known and novel driver events in T-cell acute lymphoblastic leukemia. PLoS Genet 9: e100399716. doi:10.1371/journal.pgen.1003997

Kantarjian H, Thomas D, O'Brien S, Cortes J, Giles F, Jeha S, Bueso-Ramos CE, Pierce S, Shan J, Koller C, et al. 2004. Long-term follow-up results of hyperfractionated cyclophosphamide, vincristine, doxorubicin, and dexamethasone (Hyper-CVAD), a dose-intensive regimen, in adult acute lymphocytic leukemia. Cancer 101: 2788-2801. doi:10.1002/cncr.20668

Kiel MJ, Velusamy T, Rolland D, Sahasrabuddhe AA, Chung F, Bailey NG, Schrader A, Li B, Li JZ, Ozel AB, et al. 2014. Integrated genomic sequencing reveals mutational landscape of T-cell prolymphocytic leukemia. Blood 124: 1460-1472. doi:10.1182/blood-2014-03-559542

Li G, Waite E, Wolfson J. 2017. T-cell prolymphocytic leukemia in an adolescent with ataxia-telangiectasia: novel approach with a JAK3 inhibitor (tofacitinib). Blood Adv 1: 2724-2728. doi:10.1182/bloodadvan ces.2017010470

Liu Y, Easton J, Shao Y, Maciaszek J, Wang Z, Wilkinson MR, McCastlain K, Edmonson M, Pounds SB, Shi L, et al. 2017. The genomic landscape of pediatric and young adult T-lineage acute lymphoblastic leukemia. Nat Genet 49: 1211-1218. doi:10.1038/ng.3909

López C, Bergmann AK, Paul U, Murga Penas EM, Nagel I, Betts MJ, Johansson P, Ritgen M, Baumann T, Aymerich $M$, et al. 2016. Genes encoding members of the JAK-STAT pathway or epigenetic regulators are recurrently mutated in T-cell prolymphocytic leukaemia. Br J Haematol 173: 265-273. doi:10.1111/ bjh.13952

Losdyck E, Hornakova T, Springuel L, Degryse S, Gielen O, Cools J, Constantinescu SN, Flex E, Tartaglia M, Renauld J-C, et al. 2015. Distinct acute lymphoblastic leukemia (ALL)-associated janus kinase 3 (JAK3) 
mutants exhibit different cytokine-receptor requirements and JAK inhibitor specificities. J Biol Chem 290: 29022-29034. doi:10.1074/jbc.M115.670224

Marks DI, Rowntree C. 2017. Management of adults with T-cell lymphoblastic leukemia. Blood 129: 11341142. doi:10.1182/blood-2016-07-692608

Meyer DM, Jesson MI, Li X, Elrick MM, Funckes-Shippy CL, Warner JD, Gross CJ, Dowty ME, Ramaiah SK, Hirsch JL, et al. 2010. Anti-inflammatory activity and neutrophil reductions mediated by the JAK1/ JAK3 inhibitor, CP-690,550, in rat adjuvant-induced arthritis. J Inflamm (Lond) 7: 41. doi:10.1186/14769255-7-41

Montillo M, Tedeschi A, Centurioni R, Leoni P. 2009. Treatment of relapsed adult acute lymphoblastic leukemia with fludarabine and cytosine arabinoside followed by granulocyte colony-stimulating factor (FLAGGCSF). Leukemia Lymphoma 25: 579-583. doi:10.3109/10428199709039047

Numan Y, Alfayez M, Maiti A, Alvarado Y, Jabbour EJ, Ferrajoli A, Konoplev SN, Kantarjian HM, Bose P. 2018. First report of clinical response to venetoclax in early T-cell precursor acute lymphoblastic leukemia. JCO Precis Oncol 2: 1-6. doi:10.1200/PO.18.00127

Peirs S, Matthijssens F, Goossens S, Van de Walle I, Ruggero K, de Bock CE, Degryse S, Canté-Barrett K, Briot D, Clappier E, et al. 2014. ABT-199 mediated inhibition of BCL-2 as a novel therapeutic strategy in T-cell acute lymphoblastic leukemia. Blood 124: 3738-3747. doi:10.1182/blood-2014-05-574566

Pesu M, Candotti F, Husa M, Hofmann SR, Notarangelo LD, O'Shea JJ. 2005. Jak3, severe combined immunodeficiency, and a new class of immunosuppressive drugs. Immunol Rev 203: 127-142. doi:10.1111/J .0105-2896.2005.00220.x

Rahmat LT, Nguyen A, Abdulhaq H, Prakash S, Logan AC, Mannis GN. 2018. Venetoclax in combination with decitabine for relapsed T-cell acute lymphoblastic leukemia after allogeneic hematopoietic cell transplant. Case Rep Hematol 2018: 1-4. doi:10.1155/2018/6092646

Stengel A, Kern W, Zenger M, Perglerová K, Schnittger S, Haferlach T, Haferlach C. 2015. Genetic characterization of T-PLL reveals two major biologic subgroups and JAK3 mutations as prognostic marker. Genes Chromosomes Cancer 55: 82-94. doi:10.1002/gcc.22313

Steven Martinez G, Ross JA, Kirken RA. 2016. Transforming mutations of Jak3 (A573V and M511I) show differential sensitivity to selective Jak3 inhibitors. Clin Cancer Drugs 3: 131-137. doi:10.2174/ $2212697 X 03666160610085943$

Thoma G, Drückes P, Zerwes H-G. 2014. Selective inhibitors of the Janus kinase Jak3—are they effective? Bioorg Med Chem Lett 24: 4617-4621. doi:10.1016/j.bmcl.2014.08.046

Vicente C, Schwab C, Broux M, Geerdens E, Degryse S, Demeyer S, Lahortiga I, Elliott A, Chilton L, La Starza R, et al. 2015. Targeted sequencing identifies associations between IL7R-JAK mutations and epigenetic modulators in T-cell acute lymphoblastic leukemia. Haematologica 100: 1301-1310. doi:10.3324/haematol .2015.130179

Waibel M, Gregory G, Shortt J, Johnstone RW. 2014. Rational combination therapies targeting survival signaling in aggressive B-cell leukemia/lymphoma. Curr Opin Hematol 21: 297-308. doi:10.1097/MOH 0000000000000045

Yamashita Y, Yuan J, Suetake I, Suzuki H, Ishikawa Y, Choi YL, Ueno T, Soda M, Hamada T, Haruta H, et al. 2010. Array-based genomic resequencing of human leukemia. Oncogene 29: 3723-3731. doi:10.1038/onc .2010 .117

Yin C, Sandoval C, Baeg G-H. 2014. Identification of mutant alleles of JAK3 in pediatric patients with acute lymphoblastic leukemia. Leukemia Lymphoma 56: 1502-1506. doi:10.3109/10428194.2014.957204

Zhang J, Ding L, Holmfeldt L, Wu G, Heatley SL, Payne-Turner D, Easton J, Chen X, Wang J, Rusch M, et al. 2012. The genetic basis of early T-cell precursor acute lymphoblastic leukaemia. Nature 481: 157-163. doi:10.1038/nature10725 


\section{COLD SPRING HARBOR Molecular Case Studies}

\section{Failure of tofacitinib to achieve an objective response in a DDX3X-MLLT10 T-lymphoblastic leukemia with activating JAK3 mutations}

Jonathan Wong, Meaghan Wall, Gregory Philip Corboy, et al.

Cold Spring Harb Mol Case Stud 2020, 6: a004994

Access the most recent version at doi: $10.1101 / \mathrm{mcs} . a 004994$

Supplementary http://molecularcasestudies.cshlp.org/content/suppl/2020/08/25/mcs.a004994.D
Material

References This article cites 44 articles, 15 of which can be accessed free at: http://molecularcasestudies.cshlp.org/content/6/4/a004994.full.html\#ref-list-1

License This article is distributed under the terms of the Creative Commons Attribution-NonCommercial License, which permits reuse and redistribution, except for commercial purposes, provided that the original author and source are credited.

Email Alerting Receive free email alerts when new articles cite this article - sign up in the box at the Service top right corner of the article or click here. 